\title{
COMPARATIVE STUDY BETWEEN BISAP SCORE AND RANSON SCORE IN PREDICTING SEVERITY OF ACUTE PANCREATITIS
}

\section{General Surgery}

Dr. Kumari Pallavi

M.B.B.S, M.S. (Gen. Surg.), Senior Resident, Department of General Surgery, Govt. Medical College and Hospital, Bettiah, W. Champaran, Bihar.

Dr. M. K. Nathani* M.B.B.S, M.S. (Gen. Surg.), Assistant Professor, Department of General Surgery, Govt. Medical College and Hospital, Bettiah, W. Champaran, Bihar.*Corresponding Author

Dr. C. M. Narayan M.B.B.S, M.S. (Gen. Surg.), Associate Professor, Department of General Surgery, Govt. Medical College and Hospital, Bettiah, W. Champaran, Bihar.

Dr. Debarshi Jana

Young Scientist (DST) Institute of Post-Graduate Medical Education and Research, A.J.C. Bose Road, Kolkata-700020, West Bengal, India

\section{ABSTRACT}

BACKGROUND: Acute pancreatitis has widely variable clinical and systemic manifestations spanning the spectrum from a mild, self-limiting episode of epigastric pain to severe, life-threatening, multiorgan failure. Since the morbidity and mortality of Acute Pancreatitis differ markedly between mild and severe disease (mild $<5 \%$ vs severe 20-25\%), it is very important to assess severity as early as possible. Various scoring systems like APACHE II scoring, RANSON scoring and BISAP have been used to asses Severity in Acute Pancreatitis. AIM AND OBJECTIVE: To assess the accuracy of BISAP scoring system vs RANSON scoring system in predicting Severity in an attack of acute pancreatitis. MATERIALS AND METHODS: In this study, 60 in-patients presenting with features of acute pancreatitis at Department of Surgery, Govt. Medical College and Hospital, Bettiah, W. Champaran, Bihar from April 2019 to March 2020 had been studied. It was a perspective and a retro prospective study. BISAP score and Ranson's score was calculated in all such patients based on data obtained within 48 hours of hospitalization. RESULTS: According to Atlanta Revised criteria, 30 patients had mild pancreatitis, 20 patients had moderately severe pancreatitis, 10 patients had severe pancreatitis. Of the 60 patients, 37 patients had Ranson's score less than or equal to 3.23 patients had a score of more than 3. Of the 60 patients, 39 patients had a BISAP score less than or equal to 3,21 patients had a score more than 3. CONCLUSION: From this study, we can conclude that the BISAP scoring system is not inferior to Ranson's scoring system in predicting the severity of acute pancreatitis. BISAP scoring system is very simple, cheap, easy to remember and calculate. BISAP scoring system accurately predicts the outcome in patients with acute pancreatitis. Moreover, the values in BISAP score are instantaneous and there is no time delay. Ranson's score takes a minimum of 24 hours.

\section{KEYWORDS}

Acute Pancreatitis, BISAP Score, Ranson Score, Sepsis

\section{INTRODUCTION}

Acute pancreatitis is a sudden inflammation of the pancreas. Acute pancreatitis may be a single event; it may be recurrent, or it may progress to chronic pancreatitis are associated with high mortality, even with optimal management. Acute pancreatitis is a common entity encountered during routine surgical practice and it poses a great challenge to the treating surgeon. "Acute pancreatitis is defined as a pancreatic inflammatory process, with peripancreatic and multi-organ involvement causing multi-organ dysfunction syndrome (MODS), with increased mortality rate". Acute pancreatitis (acute hemorrhagic pancreatic necrosis) is characterized by acute inflammation and necrosis of pancreas parenchyma, focal enzymic necrosis of pancreatic fat and vessel necrosis (hemorrhage). These are produced by intrapancreatic activation of pancreatic enzymes. Lipase activation causes the necrosis of fat tissue in the pancreatic interstitium and peripancreatic spaces as well as vessel damage. Necrotic fat cells appear as shadows, contours of cells, lacking the nucleus, pink, finely granular cytoplasm. It is possible to find calcium precipitates (hematoxylinophilic). Digestion of vascular walls results in thrombosis and hemorrhage. Inflammatory infiltrate is rich in neutrophils. Due to the pancreas lacking a capsule, the inflammation and necrosis can extend to include fascial layers in the immediate vicinity of the pancreas.

\section{MATERIALAND METHODS}

In this study, 60 in-patients presenting with features of acute pancreatitis at Surgery Department of Govt. Medical College and Hospital, Bettiah, W. Champaran, Bihar from April 2019 to March 2020 had been studied. It was a perspective and a retro prospective study. BISAP score and Ranson's score was calculated in all such patients based on data obtained within 48 hours of hospitalization. Acute pancreatitis was defined as 2 or more ofthe following: Characteristic abdominal pain, Increased levels of Serum amylase and/or lipase 3 times the normal value. Ultrasonography of the abdomen within the first 7 days of hospitalization demonstrating changes consistent with acute pancreatitis BISAP score and Ranson's score was calculated in all such patients based on data obtained within 48 hours of hospitalization. ACT or MRI or USG of the abdomen, obtained at any time in the first 7 days of hospitalization, was required to differentiate necrotizing from interstitial pancreatitis. Organ failure was defined as a score of $\geq 2$ in one or more of the three (respiratory, renal and cardiovascular) out of the five organ systems initially described in the Marshall score. Organ failure scores were calculated for all patients during the first 72 hours of hospitalization based on the most extreme laboratory value or clinical measurement during each 24 hours period. Duration of organ failure was defined as transient $(\leq 48$ hours) or persistent ( $\geq 48$ hours) from the time of presentation.

Inclusion criteria: Patients with history and clinical findings suggestive of acute pancreatitis with evidence of bulky edematous pancreas on USG/CT abdomen.

Exclusion criteria: Chronic pancreatitis, Acute on chronic pancreatitis.

\section{Statistical analysis}

Independent t-test was used to examine differences in age; Fischer's exact test for sex; and chi-square test for etiology were used. Sensitivity, specificity, positive predictive value, negative predictive value, and accuracy were calculated. A "p" value of less than 0.05 was considered to be statistically significant. Data analysis was performed using SPSS software.

\section{RESULTS}

According to Atlanta Revised criteria, 30 patients had mild pancreatitis, 20 patients had moderately severe pancreatitis, 10 patients had severe pancreatitis. Of the 60 patients, 37 patients had Ranson's score less than or equal to 3.23 patients had a score of more than 3. Of the 60 patients, 39 patients had a BISAP score less than or equal to 3,21 patients had a score more than 3 .

Table - 1showsRanson's score of greater than or equal to 4 predicted $93 \%$ of severe attacks and $96 \%$ of mild attacks with a positive predictive value of 93.33 and negative predictive value of 96 and accuracy of 95. Ranson's score of greater than or equal to 3 predicted more number of severe attacks $(100 \%)$ but less number of mild attacks 
(56\%) with a PPV and NPV of 100 and accuracy of 72.5. Ranson's score of greater than or equal to 5 predicted less number of severe attacks $(53 \%)$ and predicted more severe attacks as mild attacks. Ranson's score of greater than or equal to 4 had the best sensitivity, specificity, and accuracy.

Table 1 : Prediction of severity by Ranson's score

\begin{tabular}{|c|c|c|c|c|c|}
\hline $\begin{array}{c}\text { Ranson's } \\
\text { score }\end{array}$ & Sensitivity Specificity & $\begin{array}{c}\text { Positive } \\
\text { predictive } \\
\text { value }\end{array}$ & $\begin{array}{c}\text { Negative } \\
\text { predictive } \\
\text { value }\end{array}$ & Accuracy \\
\hline$\geq 3$ & 100 & 56 & 57.69 & 100 & 72.5 \\
\hline$\geq 4$ & 93.33 & 96 & 93.33 & 96 & 95 \\
\hline$\geq 5$ & 53.33 & 100 & 100 & 78.1 & 82.5 \\
\hline
\end{tabular}

Table : 2 Prediction of severity by BISAP score

\begin{tabular}{|c|c|c|c|c|c|}
\hline $\begin{array}{c}\text { BISAP } \\
\text { score }\end{array}$ & Sensitivity & Specificity & $\begin{array}{c}\text { Positive } \\
\text { predictive } \\
\text { value }\end{array}$ & $\begin{array}{c}\text { Negative } \\
\text { predictive } \\
\text { value }\end{array}$ & Accuracy \\
\hline$\leq 3$ & 93.33 & 96 & 93.33 & 96 & 95 \\
\hline$>4$ & 86.66 & 100 & 100 & 92.6 & 95 \\
\hline
\end{tabular}

Table: 3 Prediction of major organ failure and pancreatic collection by Ranson's score

\begin{tabular}{|l|l|l|l|l|l|}
\hline Ranson's score & Sensitivity & Specificity & PPV & NPV & Accuracy \\
\hline Pancreatic collection & 93.33 & 96 & 93.33 & 96 & 95 \\
\hline Major organ failure & 100 & 64.1 & 6.66 & 100 & 65 \\
\hline
\end{tabular}

Table: 4 Prediction of major organ failure and pancreatic collection by BISAP score

\begin{tabular}{|l|l|l|l|l|l|}
\hline BISAP score & Sensitivity & Specificity & PPV & NPV & Accuracy \\
\hline Pancreatic collection & 93.33 & 64.1 & 93.33 & 96 & 95 \\
\hline Major organ failure & 100 & 641 & 6.66 & 100 & 65 \\
\hline
\end{tabular}

Table: 5 Prediction of severity by Ranson and BISAP scoring systems

\begin{tabular}{|l|l|l|l|l|l|}
\hline & Sensitivity & Specificity & PPV & NPV & Accuracy \\
\hline Ranson's score & 93.33 & 96 & 93.33 & 96 & 95 \\
\hline BISAP score & 93.33 & 96 & 93.33 & 96 & 95 \\
\hline
\end{tabular}

Table - 2 shows BISAP score of less than or equal to 3 predicted $93.33 \%$ of severe attacks and $96 \%$ of mild attacks with a PPV of 93.33 and NPV of 96 and accuracy of 95 . BISAP score of less than or equal to 3 had the best sensitivity, specificity, and accuracy.

Table - 3 shows Ranson's scores were very sensitive for prediction of systemic complications $(100 \%)$ but less sensitive for prediction of local complications (93.33). BISAP score was a more accurate prediction of systemic complications $(100 \%)$ but less sensitive for prediction of local complications (93.33) as per Table -4 .

As a sensitivity, specificity, positive predictive value, negative predictive value, and accuracy were found to be the same for Ranson's and BISAP scores, BISAP scoring system is equally efficacious as Ranson scoring system in predicting the severity of acute pancreatitis (Table-5).

\section{DISCUSSION}

Multi-organ dysfunction syndrome, the extent of pancreatic necrosis, infection and sepsis are the major determinants of mortality in acute pancreatitis. Pancreatic necrosis is considered as a potential risk for infection, which represents the primary cause of late mortality. The occurrence of acute respiratory (ARF), cardiovascular (CVF) and renal failures (RF) can predict the fatal outcome in severe acute pancreatitis (SAP). A wide range of mortality $(20 \%-60 \%)$ has been reported in sap. Early diagnosis and prognostic evaluation are extremely important and may reduce the morbidity and mortality associated with SAP. On account of differences in outcome between patients with mild and severe disease, it is important to define that group of patients who will develop severe pancreatitis, predicting which still represents a challenge for the clinician. Interestingly, when seeking medical attention (usually 12 to 24 hours after the onset of pain) most patients do not exhibit multiple organ dysfunction, which is likely to emerge by the second or third day. Most patients of acute pancreatitis recover without complications, the overall mortality rate of this illness is between 2-5\%. Multiple risk stratification tools for acute pancreatitis have been developed, but their clinical usefulness is limited. Older measures and modified Glasgow score use data that are not routinely collected at the time of hospitalization. In addition, both require 48 hours, thereby missing potentially valuable early therapeutic window4. The APACHE II score is the most widely used prediction system currently but it requires the collection of a large number of parameters. APACHE II was originally developed as an intensive care instrument and requires the collection of a large number of parameters, some of which may not be relevant to prognosis". For this purpose a simple and accurate clinical scoring system that is bedside index for severity in acute pancreatitis (BISAP) scoring system was developed. This scoring system used for stratifying patients according to their risk of hospital mortality and is able to identify patients at increased risk of mortality prior to the onset of organ failure. Data or BISAP score collected within the first $24 \mathrm{hr}$ of hospitalization. Ranson score was calculated within 48 hours. The ability to stratify patients early in their course is a major step to improving management strategies in acute pancreatitis. Out of 60 patients, 38 patients had mild pancreatitis $(63.33 \%)$. Majority of patients, the disease was self-limiting. 22 patients had severe pancreatitis $(27.7 \%)$. Among 60 patients in our study, 55(91\%) were males and 5(9\%) were females. However, it was found that there was male predominance when stratifying mortality on the basis of sex in severe acute pancreatitis BISAP scores more than 3 . With respect to etiological factors of acute pancreatitis, we found alcohol being the most common cause of acute pancreatitis. The proportion of two main causes greatly depends on the geographical and cultural variations. Alcohol is the main cause in the United States of America and Finland, gallstones in southern Europe, whereas central and northern Europe sees a similar frequency of the two factors or a predominance of alcohol. In our study, out of 60 patients, $55(90 \%)$ had no organ failure, $6(10 \%)$ patients developed organ failure. Out of 6 patients, $3(50 \%)$ patients had transient organ failure and $3(50 \%)$ had persistent organ failure. Mortality was seen in 3 patients, who presented with persistent organ failure. According to a recent study, the mortality rates among severe acute pancreatitis patients have decreased from $50-58 \%$ in $1978-1982$ to $12-18 \%$ in $1993-1997$. The overall mortality in our study was $5 \%$ which is similar compared to other studies. Both Ranson and BISAP were equal in predicting the severity of acute pancreatitis. Both were equally efficacious in assessing the predictability of organ failure.

\section{CONCLUSION}

From this study, we can conclude that the BISAP scoring system is not inferior to Ranson's scoring system in predicting the severity of acute pancreatitis. BISAP scoring system is very simple, cheap, easy to remember and calculate. BISAP scoring system accurately predicts the outcome in patients with acute pancreatitis. Moreover, the values in BISAP score are instantaneous and there is no time delay. Ranson's score takes a minimum of 24 hours.

\section{REFERENCES}

Angelini G, Cavallini G, Pederzoli P, et al. Long-term outcome of acute pancreatitis: Prospective study with 118 patients. Digestion, 1993; $54: 143-147$

2. Banks P. Acute and chronic pancreatitis. In: Feldman M, Scharschmidt B, Sleisenger M, eds. trointestinal and Liver Disease 6th edition, Saunders, Philadelphia, PA, 1998, p. 809-862.

3. Banks PA. Practice guidelines in acute pancreatitis. Am J Gastroenterol., 1997; 92: 377 386

4. Beger HG, Rau B, Mayer J, Pralle U. Natural course of acute pancreatitis. World J Surg., 1997; $21: 130-135$

Beger HG. Surgical management of necrotizing pancreatitis. SurgClin North Am., 1989; 69: $11-14$.

6. Bradley EL 3rd. A clinically based classification system for acute pancreatitis. Summary of the International Symposium on Acute Pancreatitis, Atlanta, Ga, September 11 through 13, 1992. Arch., 1993; 128:586-590.

Bradley EL. A clinical based classification system for acute pancreatitis. Arch Surg., 1993; $128: 586590$

8. De Beaux AC, Palmer KR, Carter DC. Factors influencing morbidity and mortality in acute pancreatitis; an analysis of 279 cases. Gut, 1995; 37: 121-26.

9. Fagenholz PJ, Castillo CF, Harris NS, et al. Increasing United States hospital admission for acute pancreatitis, 1988-2003. Ann Epidermiol., 2007; 17: 491-7.

10. Forsmark CE. The clinical problem of biliary acute necrotizing pancreatitis: epidemiology, pathophysiology, and diagnosis of biliary necrotizing pancreatitis. J Gastrointest Surg., 2001; 5(3): 235-9.

11. Isenmann R, Beger HG. A natural history of acute pancreatitis and the role of infection. Best Practice \& Research in ClinGastroenterol., 1999; 13:291 -301

12. Larvin M, McMahon MJ. APACHE-II score for assessment and monitoring of acute pancreatitis. Lancet, 1989; 2: 201-5.

13. Levitt MD, Eckfeldt JH. Diagnosis of acute pancreatitis. In: Go V, Dimango E, Gardne J, et al., eds. The Pancreas: Biology, Pathophysiology and Disease 2nd edition. Raven Press, NY, 1993, p. 613-635.

14. Marshall JC, Cook DJ, Christou NV, et al. Multiple organ dysfunction Score: a reliable descriptor of a complex clinical outcome. Crit Care Med , 1995;23:1638-1639.

15. Mergener K, Baillie J. Acute pancreatitis. BMJ, 1998; 316: 44-48.

16. PA, Freeman ML. Practice guidelines in acute pancreatitis. Am J Gastroenterol., 2006; 101:2379-400-407

17. Ranson JH, Rifkind KM, Roses DF, et al. Objective early identification of severe acute pancreatitis. Am J Gastroenterol., 1974; 61: 443-51. 